\title{
Imaging the Molecular Dimensions and Oligomerization of Proteins at Liquid/Solid Interfaces
}

\author{
Mark J. Waner, ${ }^{\dagger}$ Martha Gilchrist, ${ }^{\dagger}$ Melvin Schindler, ${ }^{\ddagger}$ and Marcos Dantus ${ }^{*} \dagger$ \\ Departments of Chemistry and Biochemistry, Michigan State University, East Lansing, Michigan 48824
}

Received: October 2, 1997; In Final Form: December 10, 1997

\begin{abstract}
Individual Concanavalin A (ConA) molecules have been imaged at the liquid/solid interface with an atomic force microscope (AFM). Three-dimensional sizing with very high resolution $(<5 \AA)$ has been obtained by a novel approach based on height distributions, which avoids the tip convolution effects which normally affect scanning probe microscopy techniques. Each height measurement correlates to a particular molecular orientation on the surface. A large number of such measurements provide a statistical ensemble of orientations. The complete height distribution reflects the three-dimensional size of the protein sample and hence its tertiary and quaternary structure. A surface adsorption and orientation model, based on a minimization of surface adsorption energy, is proposed. This model is in good agreement with the observed height distribution of Con A molecules at the liquid/solid interface. Analysis of Con A and succinylated Con A molecules on mica demonstrates that Con A dimers are the prevalent species at the liquid/solid interface. This is in contrast to the tetrameric organization of Con A normally observed in solution. The new possibilities opened by height distribution analysis on the physical characterization of biomolecules at interfaces are also discussed.
\end{abstract}

\section{Introduction}

The behavior of proteins at the solid/liquid interface has been of great interest because of the fundamental role that membranes, cytoskeletons, and other interfaces play in cells, as well as for the more practical aspects related to the development of biological assays, biocompatibility of materials, and protein processing. ${ }^{1-3}$ The number of techniques available to quantitatively measure structure and structural changes of individual proteins at interfaces, however, is very limited. In this paper we present very high-resolution $(<5 \AA)$ three-dimensional measurements of Concanavalin A (Con A) molecules at the water/mica interface. The measurement of the molecular size allows us to determine the oligomerization state and an estimate of the surface adsorption energy for different protein orientations at the interface.

The adsorption of a protein on a solid surface is a complex process. $^{4,5}$ Current models identify two steps in the adsorption process. The first step involves the collision of the protein, which is translating by Brownian motion, with the surface. If the energetic benefit of the removal of surface adsorbed water is greater than the thermal energy of the protein molecule, then it remains on the surface; otherwise, it returns to solution. ${ }^{5}$ Once the protein is adsorbed, the second step is thought to involve a conformational change that maximizes electrostatic interactions, local interactions between electron donor moieties in the protein with electron acceptors on the surface, and maximization of van der Waals attractions. ${ }^{6}$ It is often assumed that this second step involves changes in the protein structure, akin to denaturation. There is now some experimental evidence that "compact" globular proteins maintain their native structure when adsorbed and that only some "soft/flexible" proteins suffer some degree of denaturation upon adsorption, especially on hydrophobic surfaces. $^{7}$ The second step, therefore, involves primarily an

* Corresponding author. e-mail: dantus@cem.msu.edu.

$\dagger$ Department of Chemistry.

$\doteqdot$ Department of Biochemistry. orientation process that is thermodynamically driven and yields proteins in the most energetically favorable orientation. Independent evidence of protein orientation at interfaces has been obtained by Lee and Saavedra. ${ }^{8}$

Since its recent introduction, ${ }^{9}$ atomic force microscopy (AFM) has been extensively used for imaging biological molecules. ${ }^{10-14}$ The AFM technique allows the examination of biological specimens without the need for chemical fixation/dehydration or growth of crystals, which is normally associated with other high-resolution techniques. ${ }^{10,15,16}$ In fact, samples may be imaged with AFM under liquids at physiological concentrations and $\mathrm{pH} .{ }^{17}$ A number of groups have utilized the high resolution $(\sim 1 \AA)$ of the AFM for examination of $2 \mathrm{D}$ crystallization of protein molecules. ${ }^{11,18-21}$ Others, especially Hansma, Bustamante, and Henderson, have studied isolated proteins ${ }^{17,22-28}$ or DNA molecules ${ }^{12,29-33}$ and their complexes. ${ }^{34}$ Recently, AFM has been used by the Marchant group to obtain valuable structural information for the multimeric Von Willebrand factor (MW $\sim 260 \mathrm{kD}$, monomer). ${ }^{25,35,37}$

In the case of smaller globular proteins and other small threedimensional structures, however, AFM has been limited by its inherent low resolution $(10-20 \mathrm{~nm})$ in lateral measurements. The difference between height and lateral resolution $(\sim 1$ vs $\sim 100 \AA$, respectively) is due to finite size of the probe tip $(\sim 10-50 \mathrm{~nm}$ radius of curvature) and the sample being examined $^{36-39}$ (see Figure 1A). This "convolution" effect occurs regardless of imaging conditions (vacuum, ambient, or under liquid). Some groups have proposed making sharper probe tips to reduce this problem, ${ }^{36}$ while others have examined ways to first characterize the shape of the probe tip and then mathematically "deconvolute" the AFM data. ${ }^{39}$ Because the height measurements obtained by AFM are unaffected by the size of the probe, resolution of $1 \AA$ or less can be realized in this dimension.

In this study, we extend the utility of AFM to the quantitative measurement of molecular dimensions with angstrom resolution 


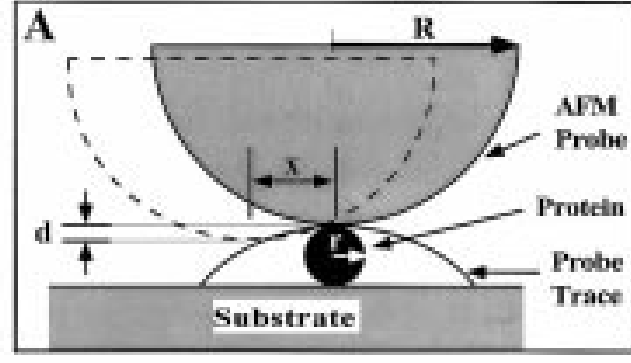

B

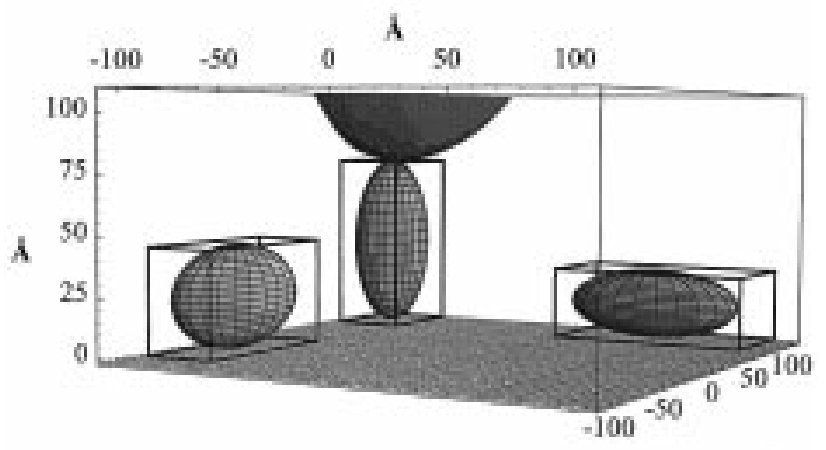

A

Figure 1. (A) Schematic representation of the AFM probe/protein interaction. Note that height measurements are not affected by tip convolution. There can be a minor discrepancy in the measured height $(d)$ when the probe of radius $(R)$ is displaced by a distance $(x)$ from the apex of the protein, of radius $(r)$. In our measurements this discrepancy amounts to a $0.5 \%$ error (see text). (B) Representative drawing of Concanavalin A dimers arranged in three orientations on a mica surface with an AFM probe positioned on top of one of the molecules. The orientation model assumes that the proteins may be crudely approximated as rectangular parallelepipeds and predicts their orientation on the surface according to the relative dimensions of the rectangular parallelepiped. The size of the molecules, the probe, and the mica lattice are drawn to scale.

at liquid/solid interfaces. Such measurements are of particular biological relevance since the preponderance of biological activities of enzymes and their binding properties occur on membrane or cytoskeletal surfaces. To avoid the loss of resolution that is normally introduced into AFM measurements as a result of probe tip geometry, we have devised a measurement strategy that measures the heights of an ensemble of individual protein molecules that have adsorbed to the atomically flat surface of mica (Figure 1B). The technique has been termed surface oriented molecular sizing (SOMS) because it utilizes the statistical thermodynamic distribution of orientations for adsorbed molecules to determine the three-dimensional structure of proteins. The use of the projected height of individual adsorbed protein molecules as the defining parameter for molecular dimensions makes this approach minimally dependent on probe tip geometry and utilizes the angstrom resolution of the AFM in the $z$ direction. Indeed, it is advantageous to use a broader tip for making these measurements, thereby minimizing the applied pressure between the tip and protein and decreasing the chance of underestimating the height of a molecule due to the digitization inherent in the microscope. Furthermore, this technique is generally applicable to contact and intermittent contact modes of imaging under ambient or liquid conditions. The relative ease, speed, and resolution with which these measurements of molecular dimension may now be performed make this technique particularly useful for examining multiple conformational equilibria of adsorbed protein subunits and the changes in shape and assembly state of multi-subunit proteins resulting from oligomerization and aggregation at liquid/solid interfaces. Such changes may be systematically examined as a function of protein concentration and ligand or allosteric activator binding. Such measurements could help to determine whether single amino acid substitutions affect protein activity as a consequence of an induced change in the oligomerization state of the mutant protein or as a result of a chemical change in the active site. We will present evidence of this phenomenon in a future publication.

We present in this article a study of Con A at the liquid/ solid interface. Our findings demonstrate that, at the water/ mica interface, Con A exists primarily as a dimer, which is in contrast to the tetrameric form which is typically observed in solution. The dimeric structure is confirmed by measurements on succinylated Con A. We also explored the possibility of denaturation at the liquid/solid interface by comparison to measurements on sodium dodecyl sulfate (SDS) treated Con A samples. Confirmation is also obtained from measurements on ferritin protein molecules, which are found to agree well with molecular dimensions derived from images obtained with X-ray diffraction and electron microscopy. The application of a surface adsorption and orientation (SAO) model in conjunction with AFM to the study of protein structure and oligomerization at the liquid/solid interface in the presence of activators, inhibitors, and denaturing agents will be discussed.

\section{Experimental Section}

Materials. Colloidal gold particles were obtained from Sigma (St. Louis, MO) and used without further purification. Three sizes of gold particles were used for the calibration: 50 $\pm 8 \AA\left(3.8 \times 10^{13}\right.$ particles $\left./ \mathrm{mL}\right), 90 \pm 12 \AA\left(6.0 \times 10^{12}\right.$ particles/mL), and $180 \pm 8 \AA\left(6.7 \times 10^{11}\right.$ particles $\left./ \mathrm{mL}\right)$. The dimensions and their deviations were determined by the manufacturer using TEM. Cationized ferritin was obtained from Polysciences (Warrington, PA) as a $20 \mathrm{mg} / \mathrm{mL}$ solution. Concanavalin A (Con A) was obtained from BoehringerMannheim (Indianapolis, IN) as lyophilized solid. Succinyl Concanavalin A was obtained from Sigma (St. Louis, MO) as a $95 \%$ protein lyophilisate. Protein purity was checked by SDS polyacrylamide gel electrophoresis. HEPES was obtained from Sigma (St. Louis, MO), and the $\mathrm{MgCl}_{2}$ was ACS grade from Columbus Chemical Industries (Columbus, $\mathrm{OH}$ ). The water used in preparing solutions was purified with a MilliQ Type I MilliPore water purification unit (19 MW resistivity). The concentrations of Con A and succinyl Con A stock solutions were determined using $A_{280}$ with an extinction coefficient of $1.37 \mathrm{~mL} \mathrm{mg}^{-1} \mathrm{~cm}^{-1} .40$

Preparation of Samples for Height Measurements with AFM. Measurements of protein height require an atomically flat substrate (less than $5 \AA$ corrugations). In this study we used muscovite mica obtained from Ward's Natural Science Establishment, Inc. (Rochester, NY.) as the substrate. In most cases we found a flatness better than $3 \AA$ over areas of tens of microns squared. When freshly cleaved, mica has a net negative surface charge. Treating a freshly cleaved surface of mica with a $5 \mathrm{mM} \mathrm{MgCl} 2$ solution results in the replacement of endogenous $\mathrm{K}^{+}$ions with $\mathrm{Mg}^{2+}$ making the surface more positively charged. The magnesium-treated surface has been demonstrated to enhance adsorption of macromolecules. ${ }^{41}$ Proteins were dissolved in $10 \mathrm{mM}$ HEPES solution at $\mathrm{pH}$ 7.3. (The concentration used in each case is specified for each protein.) Twenty-five microliters of protein solution is deposited onto the mica. Twenty minutes later, the surface is gently rinsed twice with 


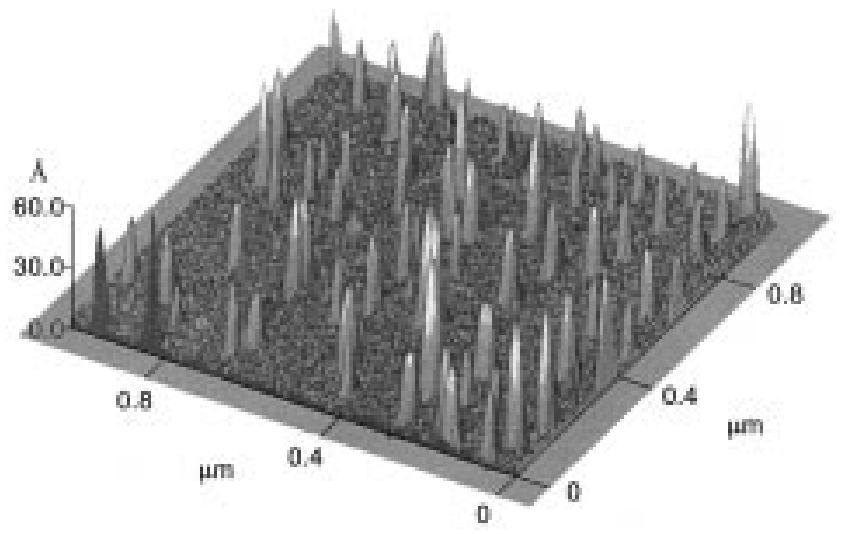

Figure 2. Typical $1 \times 1 \mu \mathrm{m}$ scan of Con A molecules on a mica substrate used to acquire height data. The sample was prepared as described in the Experimental Section. The different heights (amplified $\times 50$ ) correspond to different orientations of Con A dimers (see text).

$200 \mathrm{~mL}$ portions of MilliQ water and allowed to dry for several hours (at room temperature and 35-50\% relative humidity). This preparation yields a dispersed population of individual protein molecules when imaged by AFM (Figure 2). The concentration of protein has been chosen to provide a large population of individual protein molecules and to avoid the seeding of aggregates and monolayers. Overall, the protein coverage is always kept below $1 \%$. In the presence of a relative humidity of $\sim 30 \%$, it has been shown that there is a monolayer of water maintained on the mica surface which can act to hydrate and stabilize the adsorbed proteins. ${ }^{42}$

Atomic Force Microscopy (AFM). The microscope used for this work was an Autoprobe CP scanning probe microscope (Park Scientific Instruments), with a $5 \mu \mathrm{m}$ high-resolution scanner. Height measurements may be obtained from AFM studies carried out by contact mode or intermittent contact mode in ambient or under liquid conditions. For this study the AFM data were recorded in contact mode under controlled ambient conditions $\left(\sim 40 \%\right.$ humidity and $\left.21^{\circ} \mathrm{C}\right)$. Under these conditions a monolayer of water exists at the mica surface, ${ }^{42}$ and the protein molecules are fully hydrated. It was found that the protein molecules adhered to the mica well enough for measurements only when the humidity was less than $50 \%$, probably because high humidity increases the attractive capillary forces between probe and sample. ${ }^{12,24,42,43}$ Humidities of less than $30 \%$ were avoided to prevent protein dehydration and possible structural deformations. The effects of controlled hydration on proteins have been studied by scanning tunneling microscopy (STM). ${ }^{44,45}$ The largest changes were found to occur as the humidity dropped below $30 \%$. Unfortunately, those measurements did not provide quantitative structural information because the contrast mechanism (tunneling current) is highly dependent on the amount of solvent.

Sharpened Microlever cantilevers (Park Scientific Instruments) having a spring constant of approximately $0.05 \mathrm{~N} / \mathrm{m}$ and an integrated silicon nitride probe with a radius of curvature of approximately $20 \mathrm{~nm}$, according to the manufacturer, were used as probes. The scanner was calibrated for vertical measurements using Tobacco Mosaic Virus (cylindrical shape; diameter 18 nm) (American Type Culture Collection) and colloidal gold particles $(5 \mathrm{~nm})$ (Sigma, St. Louis). Horizontal measurements are calibrated using a two-dimensional grating with 1000 features per millimeter.

AFM images were acquired in constant force mode, in which the PZT scanner moves in the $z$ direction so as to maintain a constant force between the sample and the probe tip. To determine the minimum force to achieve stable imaging with negligible or no measurement distortion, we imaged ferritin protein molecules with a range of applied forces. At low imaging forces we accurately reproduced the well-characterized dimensions (a sphere with $120 \AA$ diameter) of this protein. ${ }^{47}$ At higher imaging forces a deformation of $\sim 10 \%$ was observed. We compared the force measurements on ferritin with similar ones on gold particles. As expected, the gold particles were more resistant to deformation due to applied pressure. The minimum force for stable imaging was found to occur within the electrostatic repulsive regime and was constant for all images. The total force applied between the tip and the cantilever under our imaging conditions contains a large attractive or capillary force $\left(\sim 10^{-9}-10^{-6} \mathrm{~N}\right.$, depending on the ambient humidity $\left.{ }^{13}\right)$ in addition to the overall repulsive force. The capillary forces between tip and sample may be minimized by imaging under liquid. These types of measurements in combination with the proposed SOMS technique may be ideally suited for the study of proteins at interfaces and are of great interest in our group.

Given that the overall accuracy of our method is limited by the roughness of the substrate to $\sim 3-5 \AA$, force distortions can be neglected except for very soft and large ( $>100 \AA$ ) features of proteins (not the case in the results presented in this article). In those cases we can use intermittent contact mode scanning techniques under liquid, where the tip-protein interaction forces can be further minimized. ${ }^{13}$ Scan size for images was $2.0 \times$ $2.0 \mu \mathrm{m}$ and $512 \times 512$ pixels at a linear scan rate of $1.5 \mathrm{~Hz}$, which is equivalent to $3 \mu \mathrm{m} / \mathrm{s}$ along the fast scan. The fairly slow linear scan rate was chosen to help minimize the lateral forces that occur between the tip and sample during the raster scanning process. The lateral forces can also be further minimized by using intermittent or noncontact modes of imaging, which are currently being investigated.

Each image of individual protein molecules contained approximately $10-50$ molecules $/ \mu \mathrm{m}^{2}$. Individual protein molecules are measured by taking the maximum height of the molecule minus the height of the local background. Each data set, consisting of more than 1000 individual height measurements, is tabulated in $3 \AA$ bins resulting in a height distribution histogram. The choice of bin size is based primarily on the substrate roughness. Our results are essentially independent of this choice. A combination of Gaussian functions is then used to fit the data. This fitting was done iteratively using constraints on the three parameters of a typical Gaussian function (i.e., center position, width, and amplitude). In all cases, the amplitudes of the functions were allowed to vary freely. The center positions of the functions were allowed to vary independently. The widths of the functions were constrained to be between 8 and $13 \AA$. The functions centered at $59 \AA$, having the lowest amplitude, have the greatest uncertainty, and their position was fixed while the other parameters were optimized. The centers of the Gaussian functions are taken as the measured molecular dimensions, while the area under the curves is proportional to the probability with which a given molecular orientation is measured. Consistency in the fitting parameters is checked by multiple repetitions of the experiments on different days. The results presented here conform to this strict criteria to less than $5 \AA$.

The final analysis consists of two different procedures: (a) determination of molecular dimensions and (b) assignment of quaternary structure (when different oligomers are observed). To assign the observed dimensions to the macromolecule, a model that takes into account the orientations of molecules at 


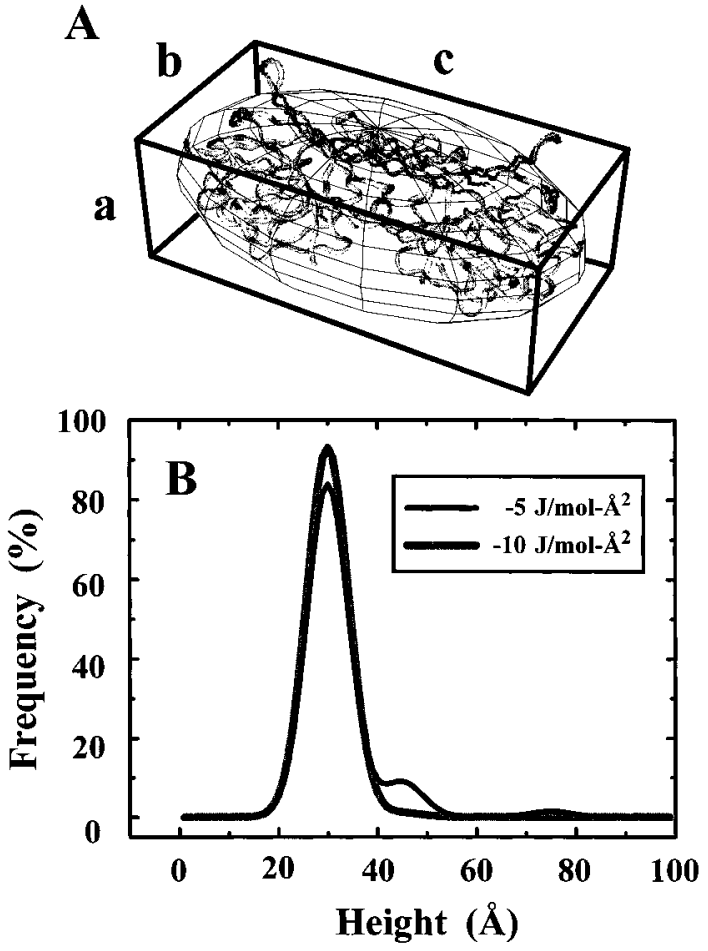

Figure 3. (A) Con A dimer (rendering based on X-ray structure) with circumscribed ellipsoid and parallelepiped of dimensions $a=30 \AA, b$ $=45 \AA$, and $c=75 \AA$. (B) Calculated height distributions for parallelepipeds, of the same dimensions as Con A dimer, as predicted by the SAO model for values of $\Delta G_{0}$ of -5 and $-10 \mathrm{~J} /\left(\mathrm{mol}^{2}\right)$. Notice that for higher protein substrate affinity proteins tend to orient so that their largest surface area lies on the mica, thereby projecting their shortest dimension perpendicular to the surface.

interfaces is required (vide infra). Protein dimensions obtained by this method are evaluated for consistency with other biophysical data available for the protein under investigation, such as molecular weight. For example, the volume of each oligomer is calculated from the assumed dimensions. Using the known molecular weight of the protein being studied, a volume is then calculated (assuming a density of $\sim 1 \mathrm{~g} / \mathrm{cm}^{3}$ ) and compared to the volume predicted by the assumed dimensions.

\section{Theory}

In the absence of chemically specific interactions of the protein molecules with the surface, the ensemble of molecules is expected to assume a thermodynamically driven distribution of orientations. A surface adsorption and orientation (SAO) model is proposed, which treats this interfacial protein orientation as an optimization of free energy.

The model assumes each protein can be enclosed by a parallelepiped of dimensions $a, b$, and $c$, where $a \leq b \leq c$ (Figure $3 \mathrm{~A}$ ). This geometrical shape is compatible with ellipsoidal models in the limit of well-rounded edges and corners. Furthermore, the measured height is independent of the degree to which the edges are rounded. A favorable interaction between the protein molecules and the hydrophilic mica surface results in orientations that maximize the surface area of contact. Therefore, a protein orientation having the $b c$ area adsorbed on the mica (where $b$ and $c$ are the largest dimensions, by definition) is considered to be the most energetically favorable, yielding a maximum probability for measured heights corresponding to dimension $a$ (see Figure 3B).

The orientation free energy after the initial adsorption step is assumed to be dependent on the protein surface in contact with the substrate. In the absence of particular surface recognition motifs, the free energy of orientation for each surface is given by

$$
\begin{aligned}
& \Delta G_{\text {orient }}^{b c} \cong \Delta G_{b c} A_{b c} \\
& \Delta G_{\text {orient }}^{a c} \cong \Delta G_{a c} A_{a c} \\
& \Delta G_{\text {orient }}^{a b} \cong \Delta G_{a b} A_{a b}
\end{aligned}
$$

where $\Delta G_{b c}$ is the free energy of orientation per unit area of the protein in contact with the substrate (in this case $b c$ ), and $A$ is the surface area of the side identified by the subscript.

The probability of orientation such that height $a, b$, or $c$ is measured depends on the different free energies of orientation, eqs $1-3$. These may be estimated by a Boltzmann distribution using

$$
\begin{aligned}
& P(a) \cong \frac{2 \exp \left(-\Delta G_{\text {orient }}^{\mathrm{bc}} / k T\right)}{Q} \\
& P(b) \cong \frac{2 \exp \left(-\Delta G_{\text {orient }}^{\mathrm{ac}} / k T\right)}{Q} \\
& P(c) \cong \frac{2 \exp \left(-\Delta G_{\text {orient }}^{\mathrm{ab}} / k T\right)}{Q}
\end{aligned}
$$

where the factor of 2 arises from the symmetry of the parallelepiped, $k$ is Boltzmann's constant, $T$ is the temperature, and $Q$ is the canonical partition function. These expressions are simplified by taking the ratio between these probabilities and by assuming that all orientation energies per unit area are equal (as expected in the absence of specific recognition sites in the surface or the protein) so that $\Delta G_{0} \equiv \Delta G_{b c}=\Delta G_{a c}=$ $\Delta G_{a b}$; we thus obtain

$$
\begin{aligned}
& P(a) / P(b)=\exp \left(\frac{\left[A_{\mathrm{ac}}-A_{\mathrm{bc}}\right] \Delta G_{0}}{k T}\right) \\
& P(b) / P(c)=\exp \left(\frac{\left[A_{\mathrm{ab}}-A_{\mathrm{ac}}\right] \Delta G_{0}}{k T}\right) \\
& P(a) / P(c)=\exp \left(\frac{\left[A_{\mathrm{ab}}-A_{\mathrm{bc}}\right] \Delta G_{0}}{k T}\right)
\end{aligned}
$$

where the free energies of orientation have been substituted by their corresponding expression (eqs 1,2, and 3). Given these equations, it is possible to predict the probabilities of each protein orientation on the surface given its dimensions. It is also possible to start with the experimentally observed dimensions $(a, b$ and $c)$ and their probabilities $\left(P_{a}, P_{b}\right.$, and $\left.P_{c}\right)$ and obtain a value for $\Delta G_{0}$ using eqs 7-9. The magnitude of $\Delta G_{0}$ determines the relative probabilities of measuring each of the three main dimensions of a protein. Figure 3 illustrates the theoretical height distribution for a parallelepiped, of the same dimensions as Con $\mathrm{A}$ dimer, for values of $\Delta G_{0}$ differing by only a factor of 2 .

The SAO model, therefore, provides a mathematical framework to analyze the height distribution profiles and obtain the relative amounts of each oligomeric state, e.g., monomer, dimer, tetramer (see the data analysis section). In addition to the obvious height differences for the oligomers, we observe that the probability of measuring those values varies extensively depending on the configuration. Therefore, for proteins of 


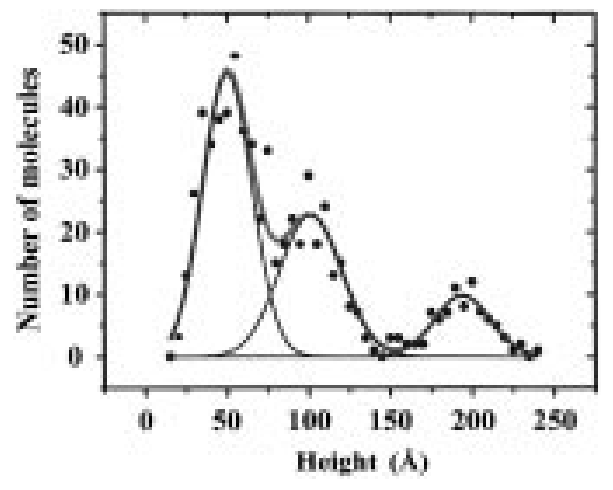

Figure 4. AFM height distribution data for colloidal gold particles adsorbed on mica. Results of the least-squares Gaussian fits to the data may be found in Table 1 .

TABLE 1: AFM Height Measurements of Colloidal Gold Particles Compared to the Values Obtained Using TEM

\begin{tabular}{ccc}
\hline $\begin{array}{c}\text { size determined } \\
\text { by TEM }(\mathrm{nm})^{a}\end{array}$ & $\begin{array}{c}\text { size determined } \\
\text { by AFM }(\mathrm{nm})\end{array}$ & $\begin{array}{c}\text { particles } \\
\text { measured }\end{array}$ \\
\hline $5.0 \pm 0.8$ & $5.0 \pm 0.8$ & 353 \\
$9.0 \pm 1.2$ & $10.1 \pm 1.6$ & 207 \\
$18.0 \pm 0.8$ & $19.4 \pm 2.0$ & 87
\end{tabular}

${ }^{a}$ TEM analysis supplied by the manufacturer.

unknown structure, this model is useful for the determination of tertiary and quaternary structure.

Most proteins are asymmetrical or oligomeric; therefore, for a given protein, different dimensions are obtained corresponding to different protein orientations (and/or oligomers) on the substrate. Given a large enough sampling, on the order of $10^{3}$ individual measurements, a distribution of measured heights is obtained that is characteristic of the molecular dimensions of the protein. To interpret the height distribution and decompose it into the dimensions of the protein, we use the SAO model to predict how molecules of specific dimensions adsorb to the substrate.

\section{Results}

Height Measurements of Colloidal Gold with AFM. Monodispersed spherical colloidal gold particles were initially employed to demonstrate the subnanometer height measurements of AFM that are minimally influenced by probe geometry. ${ }^{46}$ The particles were chosen because their sizes were comparable to that of protein molecules. Twenty five microliters of sample $\left(10^{11}-10^{13}\right.$ particles $\left./ \mathrm{mL}\right)$ was deposited onto a freshly cleaved, magnesium ion-treated $\sim 1 \mathrm{~cm}^{2}$ piece of mica and rinsed twice with $200 \mathrm{~mL}$ of MilliQ water, after a 20 min incubation time. This preparation resulted in a uniform distribution of gold particles on the mica surface. The results of height measurements on these particles are presented in Figure 4 and Table 1. The three dominant peaks in the histogram are in good agreement with the TEM analysis performed by the manufacturer. For the $18 \mathrm{~nm}$ gold we found a greater deviation presumably because some particles had slightly different shapes (nonspherical). These types of variations are more common for larger particles. In general, the similarity between the standard deviations calculated for both TEM and SOMS measurements (Table 1) suggests that the dominant source of error in these measurements may reside in the size distribution of the particles themselves rather than the measurement technique or AFM probe geometry as discussed in Materials and Methods.

Analysis of Ferritin at the Liquid/Solid Interface. To test this approach for the determination of the macromolecular

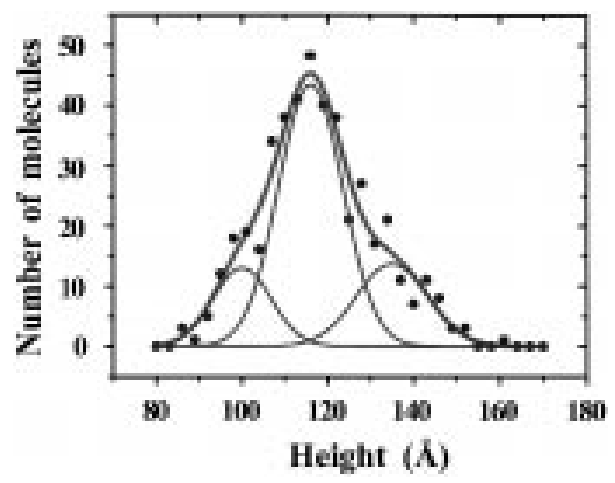

Figure 5. AFM height distribution data for ferritin. The data are best fit by three Gaussian functions centered at $97 \AA, 115 \AA$ (the intact protein), $133 \AA$ (ferritin trimers), and $144 \AA$. The two assigned peaks account for $85 \%$ of the data.

dimensions of proteins, we performed measurements on ferritin for which high-resolution structures had been previously obtained by both electron microscopy ${ }^{47}$ and X-ray crystallography. ${ }^{48}$ Ferritin (horse spleen) has been demonstrated to be a spherical molecule comprised of 24 polypeptides (ratios of homologous $\mathrm{H}$ and $\mathrm{L}$ chains) and bound iron $\left(\mathrm{Fe}^{3+}\right){ }^{47}$ Ferritin has a molecular weight of $445 \mathrm{kDa}$, and it has been shown to have a diameter of $120 \AA$ by electron microscopy ${ }^{47}$ and X-ray crystallography. ${ }^{48}$ AFM measurements of samples prepared from a $20 \mu \mathrm{g} / \mathrm{mL}$ solution of ferritin yielded a distribution of measured heights with a dominant peak at 115 $\AA$ (Figure 5), in excellent agreement with the previously reported results for ferritin. The additional peaks in the height distribution indicate the presence of ferritin trimers $(133 \AA)$ and proteins of smaller oligomeric composition. (Such ferritin structures have been previously observed with adsorbed ferritin samples on polished $\mathrm{Ti}$ and Mo using STM. ${ }^{49}$ ) Our data show the presence of three main molecular species: intact ferritin (74\%), trimers $(11 \%)$, and some smaller species at $97 \AA(9 \%)$. All other ferritin species account for $\sim 6 \%$ of the observed values.

Analysis of Concanavalin A (Con A) at the Liquid/Solid Interface. Concanavalin A (Con A) is a lectin, obtained from the jackbean (Canavalia ensiformis), that binds to mannose, glucose, and glycoconjugates containing these saccharides. ${ }^{50-52}$ The native protein is composed of four identical subunits, each with molecular weight $25500 \mathrm{kDa}^{52}$ Con $\mathrm{A}$ in solution is normally observed as a tetramer at $\mathrm{pH} 7.0$ or above. ${ }^{52}$ However, it was shown by Gordon and Young ${ }^{53}$ that Con A dissociates into dimers as a consequence of dilution and ionic strength at a concentration $\leq 5 \mu \mathrm{g} / \mathrm{mL}$ and a $\mathrm{pH} \geq 7.0$. According to X-ray diffraction studies, the molecular dimensions of the dimer are approximately $30 \AA \times 45 \AA \times 75 \AA$, while those of the tetramer are approximately $60 \AA \times 70 \AA \times 70 \AA$ (Protein DataBank, $1 \mathrm{CN} 1^{54}$ ) (Figure 7).

Preparations of Con A solutions ( $400 \mathrm{ng} / \mathrm{mL})$, as described in the Experimental Section, yielded monodispersed individual protein molecules adsorbed on the mica surface (see Figure 2, for example). Analysis of several such images results in the height distribution shown in Figure 6. This distribution is the result of 2000 individual protein molecule measurements. The height distribution is best fit by four Gaussian functions centered at $18,28,42$, and $59 \AA$ (see Table $2 \mathrm{~A}$ ). Inspection of the $\mathrm{X}$-ray structure of the Con A dimer (see Figure 7) indicates that the observed height distribution corresponds to individual Con A dimers oriented such that their largest surface area is in contact with the mica substrate, projecting the $30 \AA$ dimension perpendicular to the surface. This orientation is expected and can be understood on the basis of SAO model. The 28 and 42 


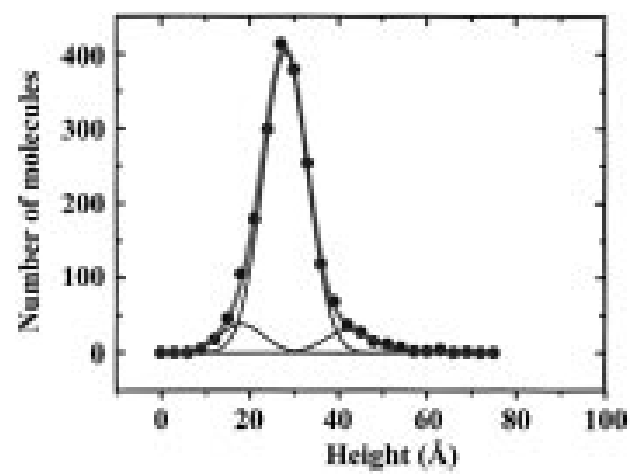

Figure 6. AFM height distribution data (2000 height measurements) for Con A The data are best fit by four Gaussian functions centered at $18,28,42$, and $59 \AA$. The results obtained from this fit may be found in Table $2 \mathrm{~A}$.

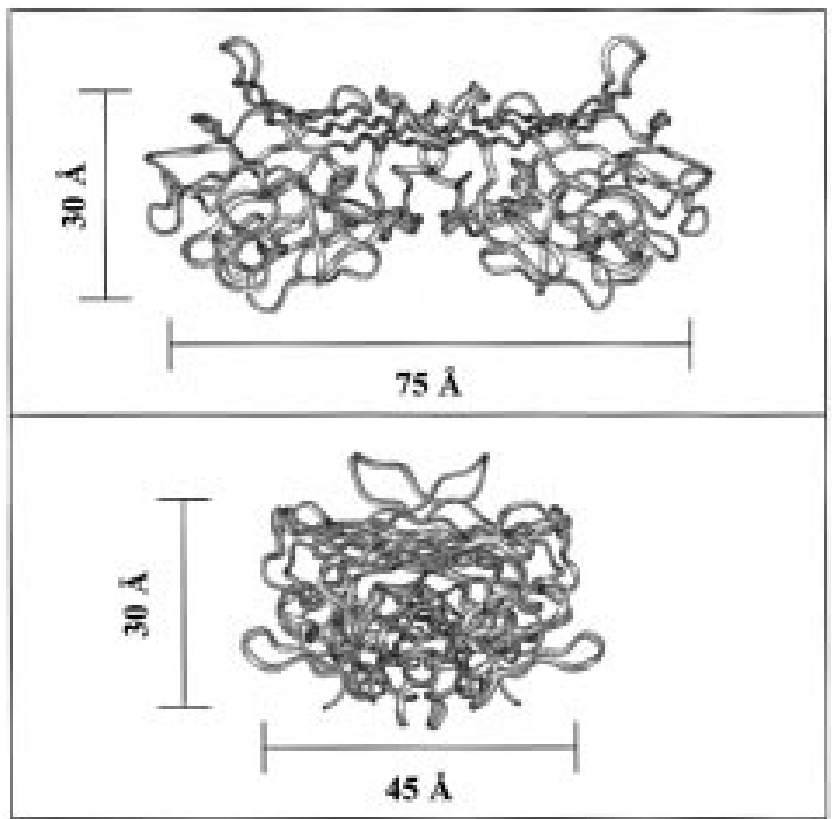

Figure 7. Ribbon rendering of the Con A dimer (Protein DataBank, $1 \mathrm{CN} 1^{54}$ ). (A) Side view showing the short axis, $30 \AA$ (which does not include the hairpin turns), and the longest axis, $75 \AA$ for end to end. (B) Side view showing the intermediate axis measuring approximately $45 \AA$ (neglecting the two hairpin turns at the extremes).

$\AA$ dimensions are in excellent agreement with the $\mathrm{X}$-ray structure (see Figure 7). The $59 \AA$ measurement is shorter than the 75 $\AA$ expected, assuming that individual Con A dimers project their longest axis away from the mica ( $0^{\circ}$ from normal inclination). This $0^{\circ}$ orientation is not favorable on the basis of protein structure shown in Figure 7; therefore, one would expect some angle of inclination. Our measurements indicate an inclination of about $38^{\circ}$ from normal. We will show that the presence of the $18 \AA$ population $(\sim 8 \%$ of the total population) can be attributed to denatured $\mathrm{Con} \mathrm{A}$ in the following experiments.

To rule out the possibility that Con A was denaturing at the mica surface, two experiments were carried out. The first one involves imaging of succinylated Con A which is known to exist only as a dimer, over a wide range of $\mathrm{pH}^{.5}$ The second experiment was to purposely denature the Con A and determine the height distribution with our technique.

Results from the succinylated Con A (1576 individual height measurements) show a very similar height distribution to that obtained from Con A (see Figure 8 and Table 2B). The three main peaks found are at 29,45 , and $59 \AA$, corresponding closely (within $3 \AA$ ) to those found for Con A. These results indicate
TABLE 2: Results from Height Distribution Analysis and Least-Squares Fit to Gaussian Functions ${ }^{a}$

\begin{tabular}{|c|c|c|c|c|}
\hline & height $(\AA)$ & fwhm $(\AA)^{b}$ & $\operatorname{area}^{c}$ & probability $(\%)^{d}$ \\
\hline \multicolumn{5}{|c|}{ (A) Concanavalin $\mathrm{A}$} \\
\hline * & 18 & 10 & 489 & \\
\hline$a$ & 28 & 10 & 5079 & 91.8 \\
\hline$b$ & 42 & 10 & 409 & 7.4 \\
\hline$c$ & 59 & 10 & 45 & 0.8 \\
\hline \multicolumn{5}{|c|}{ (B) Succinylated Con A } \\
\hline * & 19 & 9 & 1056 & \\
\hline$a$ & 29 & 13 & 1597 & 87.7 \\
\hline$b$ & 45 & 8 & 164 & 9.0 \\
\hline$c$ & 59 & 13 & 59 & 3.2 \\
\hline \multicolumn{5}{|c|}{ (C) SDS-Treated Con A } \\
\hline * & 18 & 10 & 3679 & \\
\hline$a$ & 29 & 13 & 1938 & 81.2 \\
\hline$b$ & 46 & 12 & 350 & 14.7 \\
\hline$c$ & 59 & 12 & 100 & 4.2 \\
\hline
\end{tabular}

${ }^{a}$ The values in these tables are the result of the height distribution analysis of measurements made on 2000, 1576, and 1983 individual proteins for A, B, and C, respectively. ${ }^{b}$ These values represent the full width at half-maximum (fwhm) for each of the Gaussian functions in the fit. ${ }^{c}$ These measurements correspond to the area under the Gaussian function and are proportional to the total number of molecules within a range of heights in $\AA .{ }^{d}$ The probability excludes the particles found to have a dimension of $18-19 \AA$ labeled by $*$, which are determined to arise from the presence of denatured protein molecules. The values of $\Delta G_{0}$ are the average of three measurements, using eqs 7-9 (see text). For Con A (A) and succinylated Con A (B) we obtain $-9.4 \pm$ $2.0 \mathrm{~J} /\left(\mathrm{mol} \AA^{2}\right)$ and $-6.2 \pm 0.2 \mathrm{~J} /\left(\mathrm{mol} \AA^{2}\right)$, respectively.

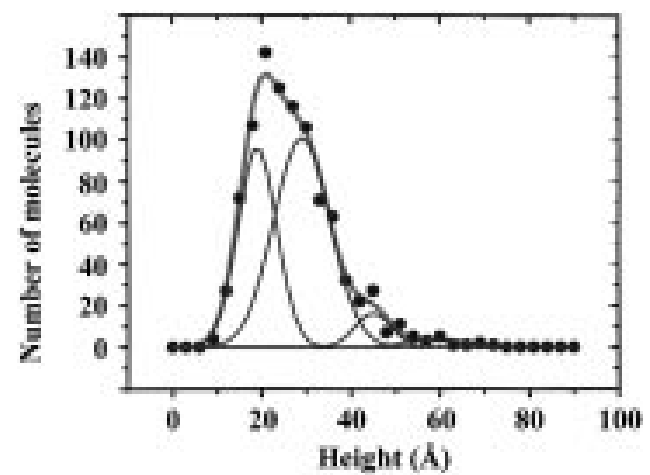

Figure 8. AFM height distribution data (1576 height measurements) for succinyl Con A. The data are best fit by four Gaussian functions centered at 19, 29, 45, and $59 \AA$. The results obtained from the leastsquares fit may be found in Table $2 \mathrm{~B}$.

that Con A exists as a dimer and not a tetramer at the water/ mica interface under these conditions. This result confirms the observations of Gordon and Young ${ }^{53}$ and provides support for the capability and sensitivity of this approach for measuring changes in the oligomerization state of proteins. The only difference is an additional population of molecules with a height of $19 \AA$. An explanation of this population with a smaller dimension is given below.

To investigate the possibility of denaturation of Con A at the water/mica interface, we treated the protein with $2 \%$ sodium dodecyl sulfate (SDS) for $2-3 \mathrm{~min}$ at $90-95^{\circ} \mathrm{C}$ prior to dilution and preparation of samples. The height distribution from the SDS-treated Con A samples, from 1983 individual height measurements, is shown in Figure 9 (see also Table 2C). Note that the major population is centered at $18 \AA$. The results indicate that $\sim 61 \%$ of the protein molecules were denatured. The rest of the distribution shows peaks at 29,46 , and $59 \AA$, in excellent agreement $(<5 \AA)$ with the previous two sets of data. This indicates that some of the Con A molecules were not 


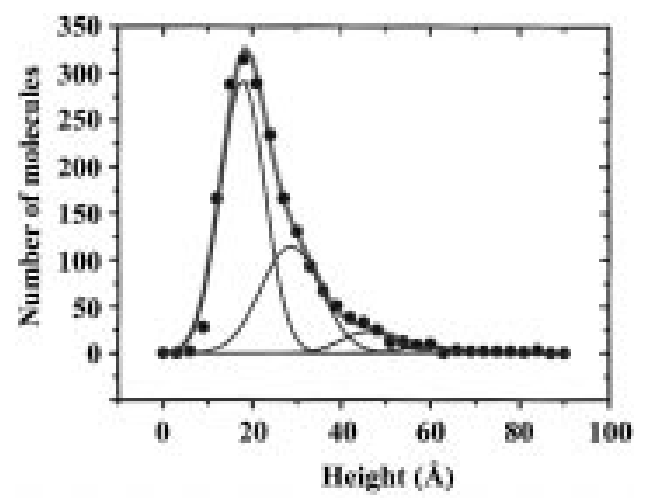

Figure 9. AFM height distribution data (1983 height measurements) for SDS-treated Con A. The data are best fit by four Gaussian functions centered at 18, 29, 46, and $59 \AA$. The results obtained from this leastsquares fit may be found in Table 2C.

denatured or underwent refolding upon dilution of the SDS during sample preparation. This result allows us to assign the peak at $19 \AA$ in the succinylated Con A samples to the presence of $\sim 37 \%$ denatured protein in the sample.

Further analysis of the results by the SAO model can be used to determine the free energy of orientation per unit area, $\Delta G_{0}$, for Con $\mathrm{A}$ and succinylated Con $\mathrm{A}$ on $\mathrm{Mg}^{2+}$-treated mica. Using eqs 7-9 and values (height and probability) from Table 2 , we calculate average values of $-9.4 \pm 2.0$ and $-6.2 \pm 0.2$ $\mathrm{kJ} /\left(\mathrm{mol} \AA^{2}\right)$, respectively. The uncertainty is the standard deviation of the three values calculated from eqs 7-9. Dayto-day variability of as much as $40 \%$ in the calculated value of $\Delta G_{0}$ has been observed. ${ }^{56}$ The approximate surface area of each face of the protein can be calculated from the measured dimensions. With these values we calculate the free energy of orientation for each of the three different orientations (see Table 2 ), the most stable of these being $\sim-20 \mathrm{~kJ} / \mathrm{mol}$ when the Con A molecules have their largest surface oriented toward the mica. These values are larger than $k T(2.5 \mathrm{~kJ} / \mathrm{mol})$, indicating this orientation is relatively stable. The free energy is small enough that distortions in the native structure are not expected. Differences in the free energy for each orientation determine the observed height distribution. The values for Con A and succinylated Con A are similar, as expected; however, we believe that there may be some small difference related to modification of amino acid side chains of Con A by the succinylation process. Succinylation involves the modification of amines in the protein with a concomitant decrease in positive charge. For Con A, an average of 10 sites per subunit are succinylated, ${ }^{55}$ causing changes in its interaction with the mica surface.

In conclusion, we have obtained angstrom resolution measurements directly from individual proteins at the liquid/solid interface. Our measurements, 28, 42, and $59 \AA$, indicate that Con A adsorbs onto mica substrates as a dimer, primarily having its smallest dimension projected normal to the surface. Our measurements are in very good agreement with the X-ray structure determined by Shoham et al. ${ }^{54}$ Although the tetrameric form of Con A is normally observed in solution, the solution from which we prepared our samples was $\sim 400 \mathrm{ng} / \mathrm{mL}$ at a $\mathrm{pH}$ $=7.3$, which according to Gordon and Young is expected to contain primarily the dimer species. ${ }^{53}$ This dimeric state is conserved upon adsorption of the Con A molecules to the mica surface. The dimeric structure was confirmed with measurements on succinylated Con A. Evidence to the orientation of the Con A dimers on the mica comes from the prevalence of $28 \AA$ measurements in the data and analysis of the different heights (42 and $59 \AA$ ) using the SAO model. The possibility of protein denaturation caused by the adsorption process was addressed by measurements on Con A treated by the denaturing agent SDS, which indicated that the degree of denaturation upon adsorption is minimal ( $<5 \AA$ changes). Applying the SAO model to our measurements results in approximate values for the free energy of orientation for these molecules. We are currently investigating the effects of large changes in the protein/ surface interaction on these values.

\section{Discussion}

Measurements of individual Concanavalin A and succinyl Con A molecules at the water/mica interface have determined that the Con A dimer is the prevalent structure. The measurements obtained $(29 \times 45 \times 59 \AA)$ for these dimers are in good agreement with the dimensions determined by X-ray diffraction studies. Further, the results for Con A, succinyl Con A, and SDS-treated ConA are self-consistent and reproducible. In addition to the structural information obtained, the proposed model also allows us to predict a value for the free energy of adsorption for Con $\mathrm{A}$ at the water/mica interface and the orientation of the adsorbed proteins.

We conservatively estimate that the accuracy of this technique is $5 \AA$ or less. This estimate is related to a number of factors. First of all, there is $3-5 \AA$ roughness in the mica substrates which we use. Second, although the ultimate resolution of the AFM in the $z$ dimension is $1 \AA$ or less, we are not optimizing our imaging conditions for single-point measurements of height; rather, we are trading accuracy for acquisition time. For the purposes of three-dimensional protein oligomerization state and orientation, the accuracy $(<5 \AA)$ is sufficient.

The technique presented here provides a means to measure the molecular dimensions for the complete size range of monomeric and oligomeric proteins adsorbed to a surface (natural or synthetic). These measurements may then be utilized to determine the oligomerization or aggregation state of the protein. This approach extends the nanometer resolution of $\mathrm{AFM}$, in the contact or intermittent contact modes under ambient or liquid conditions, to that of angstroms by avoiding excessive image broadening of biological samples that is associated with the lateral distortions introduced by the probe tip geometry. ${ }^{36-39}$ Probe-protein interactions, mainly lateral and vertical pressure, can cause the molecule to appear shorter. ${ }^{26}$ With regard to protein-substrate interaction, there is an attractive interaction between the polar surface of the protein molecule and the charged mica surface, possibly deforming the molecule and causing it to flatten against the mica surface. A study using lysozyme has shown that it retains its activity when adsorbed onto mica and imaged with AFM, so these deformations may not be extensive enough to alter biological activity. ${ }^{27}$ Our measurements confirm the conservation of native structure when compared with available X-ray data.

A thermodynamic model was derived to understand the orientation of proteins at the liquid/solid interface. Large deviations from this SAO model by adsorbed proteins would imply a preferred orientation that is presumably related to recognition between a protein domain and the surface. The extent of deviation from the orientation model may therefore be useful in defining preferred molecular orientation for oligomers or multiple equilibria for dissociated subunits of oligomers. In these cases one is able to extend the simple model presented here, by allowing each face of the protein to have a different value for $\Delta G_{0}$ (eqs $1-3$ ). In this way a quantitative value may be assigned to the energy difference associated with 
each orientation. Indeed, binding studies with streptavidin and free biotin provide strong evidence for a ligand-mediated preferential orientation for the adsorption of streptavidin dimers and tetramers to the mica surface (work in progress).

An examination of the preferred orientation of the molecules also provides the opportunity to predict the organization of individual subunits within the protein oligomer and potentially evaluate the conformational flexibility of dissociated individual subunits. Measurements with angstrom resolution may be performed on functional complexes that are associated with a surface, a more common biological interaction within and between cells. This paper presents an approach that extends the analytical power and the resolution of AFM to provide direct physical characterization (both dimensions and orientation) of biomolecules at interfaces and is minimally dependent on the geometry of the probe tip. This approach provides (1) the capability to examine the concentration- and ligand-dependent properties of protein oligomerization using picomole amounts of protein, (2) offers the capability to distinguish between the possible quaternary forms of oligomers and their interconversions resulting from the binding of ligands, substrates, or allosteric effectors, and (3) determines whether adsorption of oligomeric proteins to surfaces (natural or synthetic) can modify protein assembly and aggregation state. Such measurements can be most useful in studies of enzyme regulation, DNA/RNA organization, gene regulation, protein biosynthesis and chaperonin function, bacterial attachment and adhesion, and the effects of posttranslational modification on protein organization and activity.

Acknowledgment. This research was partially funded by the REF-Protein Structure, Function and Design Award (MSU), the Center for Fundamental Materials Research (MSU), and a Camille and Henry Dreyfus New Faculty Award (M.D.). We are very grateful to Dr. Neil Bowlby for programming the data analysis software and Drs. Leslie Kuhn, Michael Garavito, John Wang, and Jack Preiss of the Department of Biochemistry for their incisive critiques. M.D. is a Beckman Young Investigator and a Packard Science and Engineering Fellow.

\section{References and Notes}

(1) Horbett, T. A.; Brash, J. L. In Proteins at Interfaces, Physicochemical and Biochemical Studies, Brash, J. L., Horbett, T. A., Eds.; ACS Symposium Series 343; American Chemical Society: Washington, DC, 1987; p 1

(2) Norde, W. Adv. Colloid Interface Sci. 1986, 25, 267

(3) Brash, J. L.; Horbett, T. A. In Proteins at Interfaces II, Fundamentals and Applications; Horbett, T. A., Brash, J. L., Eds.; ACS Symposium Series 602; American Chemical Society: Washington, DC, 1995; p 1.

(4) Elwing, H.; Askenda, A.; Ivarsson, B.; Nilsson, U.; Welin, S.; Lundström, I. In Proteins at Interfaces, Physicochemical and Biochemical Studies; Brash, J. L., Horbett, T. A., Eds.; ACS Symposium Series 343; American Chemical Society: Washington, DC, 1987; p 468.

(5) Nadarajah, A.; Lu, C. F.; Chittur, K. K. In Proteins at Interfaces II, Fundamentals and Applications; Horbett, T. A., Brash, J. L., Eds.; ACS Symposium Series 602; American Chemical Society: Washington, DC, 1995; p 181

(6) van Oss, C. J.; Wu, W.; Giese, R. F. In Proteins at Interfaces II, Fundamentals and Applications; Horbett, T. A.; Brash, J. L., Eds.; ACS Symposium Series 602, American Chemical Society: Washington, DC, 1995; p 80.

(7) Yan, G.; Li, J.-T.; Huang, S.-C.; Caldwell, K. D. In Proteins at Interfaces II, Fundamentals and Applications; Horbett, T. A.; Brash, J. L., Eds.; ACS Symposium Series 602, American Chemical Society: Washington, DC, 1995; p 256.

(8) Lee, J. E.; Saavedra, S. S. In Proteins at Interfaces II, Fundamentals and Applications; Horbett, T. A.; Brash, J. L., Eds.; ACS Symposium Series 602; American Chemical Society: Washington, DC, 1995; p 269. 930.
(10) Lal, R.; John, S. A. Am. J. Physiol. 1994, 266, C1.

(11) Butt, H.-J.; Downing, K. H.; Hansma, P. K. Biophys. J. 1990, 58, 1473

(12) Bustamante, C.; Vesenka, J.; Tang, C. L.; Rees, W.; Guthold, M.; Keller, R. Biochemistry 1992, 31, 22.

(13) Bustamante, C.; Rivetti, C. Annu. Rev. Biophys. Biomol. Struct. 1996, 25, 395 .

(14) Louder, D. R.; Parkinson, B. A. Anal. Chem. 1994, 66, 84R.

(15) Schnyder, T.; Engel, A.; Lustig, A.; Wallimann, T. J. Biol. Chem. 1988, 263, 16954

(16) Dykstra, M. J. A Manual of Applied Techniques for Biological Electron Microscopy; Plenum: New York, 1993.

(17) Weisenhorn, A. L.; Drake, B.; Prater, C. B.; Gould, S. A. C.; Hansma, P. K.; Ohnesorge, F.; Egger, M.; Heyn, S. P.; Gaub, H. E. Biophys. J. 1990, 58,1251

(18) Schabert, F.; Hefti, A.; Goldie, K.; Stemmer, A.; Engel, A.; Meyer

E.; Overney, R.; Güntherodt, H.-J. Ultramicroscopy 1992, 42B, 1118.

(19) Schabert, F. A.; Henn, C.; Engel, A. Science 1995, 268, 92.

(20) Ohnishi, S.; Hara, M.; Furuno, T.; Sasabe, H. Biophys. J. 1992, 63,1425

(21) Malkin, A. J.; Kuznetsov, Y. G.; McPherson, A. J. Struct. Biol. 1996, 117,124

(22) Roberts, C. J.; Williams, P. M.; Davies, J.; Dawkes, A. C.; Sefton, J.; Edward, J. C.; Haymes, A. G.; Bestwick, C.; Davies, M. C.; Tendler, S. J. B. Langmuir 1995, 11, 1822 .

(23) Ill, C. R.; Keivens, V. M.; Hale, J. E.; Nakamura, K. K.; Jue, R. A.; Cheng, S.; Melcher, E. D.; Drake, B. Biophys. J. 1993, 64, 919.

(24) Edstrom, R. D.; Meinke, M. H.; Yang, X.; Yang, R.; Elings, V.; Evans, D. F. Biophys. J. 1990, 58, 1437

(25) Marchant, R. E.; Lea, A. S.; Andrade, J. D.; Bockenstedt, P. J. Colloid Interface Sci. 1992, 148, 261.

(26) Radmacher, M.; Fritz, M.; Cleveland, J. P.; Walters, D. A.; Hansma, P. K. Langmuir 1994, 10, 3809 .

(27) Radmacher, M.; Fritz, M.; Hansma, H. G.; Hansma, P. K. Science 1994, 265, 1577.

(28) Henderson, R. M.; Schneider, S.; Li, Q., L.; Hornsby, D.; White, S. J.; Oberleithner, H. Proc. Natl. Acad. Sci. U.S.A. 1996, 93, 8756.

(29) Benzanilla, M.; Manne, S.; Laney, D. E.; Lyubchenko, Y. L.; Hansma, H. G. Langmuir 1995, 11, 655.

(30) Hansma, H. G.; Sinsheimer, R. L.; Li, M. Q.; Hansma, P. K. Nucl. Acids Res. 1992, 20, 3585 .

(31) Hansma, H. G.; Vesenka, J.; Siegerist, C.; Kelderman, G.; Morret, H.; Sinsheimer, R. L.; Elings, V.; Bustamante, C.; Hansma, P. K. Science 1992, 256, 1180.

(32) Henderson, E. Nucl. Acids Res. 1992, 20, 445.

(33) Shaiu, W.-L.; Vesenka, J.; Jondle, D.; Henderson, E.; Larson, D. D. J. Vac. Sci. Technol. A 1993, 11 (4), 820.

(34) Wyman, C.; Rombel, I.; North, A. K.; Bustamante, C.; Kustu, S. Science 1997, 275, 1658.

(35) Siedlecki, C. A.; Lestini, B. J.; Kottke-Marchant, K.; Eppell, S. J.; Wilson, D. L.; Marchant, R. E. Blood 1996, 88 (8), 2939.

(36) Keller, D. J.; Franke, F. S. Surg. Sci. 1993, 294, 409.

(37) Eppell, S. J.; Zypman, F. R.; Marchant, R. E. Langmuir 1993, 9, 2281

(38) Allen, M. J.; Hud, N. V.; Balooch, M.; Tench, R. J.; Seikhaus, W. J.; Balhorn, R. Ultramicroscopy 1992, 42B, 1095.

(39) Markiewicz, P.; Goh, M. C. Langmuir 1994, 10, 5.

(40) Yariv, J.; Kalb, A. J.; Levitzski, A. Biochim. Biophys. Acta 1968, $165,303$.

(41) Apell, H. J.; Colchero, J.; Linder, A.; Marti, O. In STM and AFM in Biology; Marti, O., Amrein, M., Eds.; Academic Press: San Diego, CA 1993; p 282.

(42) Hu, J.; Xiao, X.-D.; Ogletree, D. F.; Salmeron, M. Science 1995 $268,267$.

(43) Thundat, T.; Zheng, X.-Y.; Chen, G. Y.; Warmack, R. J. Surf. Sci Lett. 1993, 294, L939.

(44) Leggett, G. J.; Davies, M. C.; Jackson, D. E.; Roberts, C. J.; Tendler, S. J. B.; Williams, P. M. J. Phys. Chem. 1993, 97, 8852.

(45) Parker, M. C.; Davies, M. C.; Tendler, S. J. B. J. Phys. Chem. 1995, 99, 16155.

(46) Height measurements of small spherical particles with AFM are slightly affected, $\sim 1 \%$, because of the geometry of the probe and because individual scan lines may be separated by distances comparable to the size of particles being measured. This error occurs when the end of the probe, which can be modeled as a hemisphere, is not exactly at the apex of the protein. For example, in a $2 \times 2 \mu \mathrm{m}$ square image digitized to $512 \times 512$ pixels, the end of the probe could be as far as $20 \AA$ from the apex of the molecule if it is located exactly between two scan lines. In Figure 1A, the idealized shapes of the end of the probe and a spherical protein molecule are illustrated. Because of the finite number of points that are recorded, the apex of the protein will usually not be exactly under the center of the probe; therefore, the measured height of the particle will be slightly smaller. The 
discrepancy, $d$, can be calculated as follows:

$$
d=(R+r)-\sqrt{(R+r)^{2}-X^{2}}
$$

where $R$ is the radius of curvature of the probe, $r$ is the radius of a spherical protein, and $X$ is the horizontal distance between the probe and the protein when the probe is closest to the protein (see Figure 1A). Probe sizes for our measurements had approximately a $24 \mathrm{~nm}$ radius of curvature, as calculated from the width and height measurements of colloidal gold particles of known dimensions ${ }^{39}$ For particles with a diameter of $40 \AA$, the maximum height deviation can be as much as $0.7 \AA$. We have modeled this effect by calculating the average error expected given 500 randomly placed 40 A diameter spheres. (Spherical geometry maximizes this resolution effect.) The average error obtained from our simulation was $0.2 \AA$, which amounts to $0.5 \%$ error in the height measurement of the particles. This error is smaller than the natural roughness of atomically flat mica; therefore, no attempt was made to minimize it or correct for it.

(47) Massover, W. H. Micron 1993, 24, 389.

(48) Harrison, P. M. J. Mol. Biol. 1963, 6, 402.
(49) Yau, S.-T.; Zhou, Y. Mod. Phys. Lett. B 1995, 9, 187.

(50) Concanavalin A as a Tool; Bittiger, H., Schnebli, H. P., Eds.; John Wiley \& Sons: New York, 1976.

(51) Sharon, N.; Lis, H. Science 1989, 246, 227.

(52) Reeke, G. N., Jr.; Becker, J. W.; Cunningham, B. A.; Wang, J. L.; Yahara, I.; Edelman, G. M. In Concanavalin A; Chowdhury, T. K., Weiss, A. K., Eds.; Plenum Publishing Corp.: New York, 1975.

(53) Gordon, J. A.; Young, R. K. J. Biol. Chem. 1979, 254, 1932

(54) Shoham M.; Yonath. A.; Sussman, J. L.; Moult, J.; Traub, W.; Kalb, A. J. J. Mol. Biol. 1979, 131, 137.

(55) Gunther, G. R.; Wang, J. L.; Yahara, I.; Cunningham, B. A.; Edelman, G. M. Proc. Natl. Acad. Sci. U.S.A. 1973, 70 (4), 1012.

(56) The origin of this variability is currently under investigation. Some of the variation may be attributed to the amount of background noise, perhaps due to differences in the mica substrate or changes in instrument performance. Capillary forces may also play a role in this variability. Further experiments, carried out under liquid conditions, will help us make this assessment 